$31-38$

\title{
A systematic review of cervical artificial disc replacement wear characteristics and durability
}

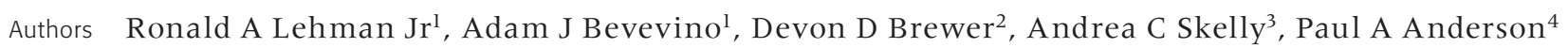

Institutions $\quad{ }^{1}$ Walter Reed National Military Medical Center, Bethesda, MD, USA

${ }^{2}$ Spectrum Research Inc, Tacoma, WA, USA. Interdisciplinary Scientific Research, Seattle WA, USA

${ }^{3}$ Spectrum Research Inc, Tacoma, WA, USA

${ }^{4}$ Department of Orthopedics \& Rehabilitation, University of Wisconsin, Madison, WI, USA

\section{ABSTRACT}

Study design: Systematic review.

Clinical questions: (1) What evidence is available from studies of cervical total disc arthroplasty (CADR) failure and retrieval regarding durability, wear, and reasons for failure of C-ADR? (2) What evidence is available from experimental models regarding the durability of C-ADR beyond 5 years?

Methods: We searched electronic databases to identify published reports of explanted cervical artificial discs and biomechanical simulations of disc wear.

Results: Nine articles were identified describing 17 devices explanted from human patients and four articles describing 23 devices explanted from non-human subjects. Wear properties were not consistently reported across studies, so summaries for specific variables are based on few cases. No device had been implanted longer than 4 years. In both human and non-human subjects, devices showed evidence of metallic and polymeric (for discs with polymer components) debris. Inflammatory cells were frequently present in surrounding soft tissues. Signs of infection were uncommon.

Four patients had reactions interpreted as hypersensitivity to metal. We identified three articles on biomechanical wear simulations. Devices were tested between 10 and 20 million cycles in axial loading, flexion/extension, and lateral bending. No device failures were reported. One study suggests such simulations may represent 50 or more years of wear in actual patients.

Conclusion: Cervical disc implants consistently produced polymeric and metallic debris, which was typically accompanied by inflammation. Hypersensitivity to metal may increase risk for device failure. Biomechanical simulations indicate that cervical disc implants may be durable beyond the currently reported length of clinical follow-up. 


\section{STUDY RATIONALE AND CONTEXT}

Cervical artificial disc replacement (C-ADR) has gained popularity and utility in the treatment of various cervical spine disorders. A growing body of literature is now available justifying its use, and as the procedure becomes more commonplace evaluating the longevity and durability of the implants is increasingly important. Just as with diarthrodial joint arthroplasties, it is critical to understand long-term wear characteristics and their effects on host tissue. However, unlike knee and hip arthroplasty, longterm follow-up on cervical artificial disc replacement is not yet available. As a result, most data on the durability of the C-ADR implants comes from human and non-human animal retrieval studies and biomechanical simulations.

\section{OBJECTIVE}

To summarize, through systematic review, data on the long-term durability of the current C-ADR implants. Focusing on human and non-human retrieval and biomechanical studies, we describe evidence on the mechanisms of failure, the wear patterns of implants, and the effects of implant wear on the surrounding tissues.

\section{CLINICAL QUESTIONS}

(1) What evidence is available from studies of C-ADR failure and retrieval regarding durability, wear and reasons for failure of C-ADR? (2) What evidence is available from experimental models regarding the durability of C-ADR beyond 5 years?

Fig 1 Results of literature search.

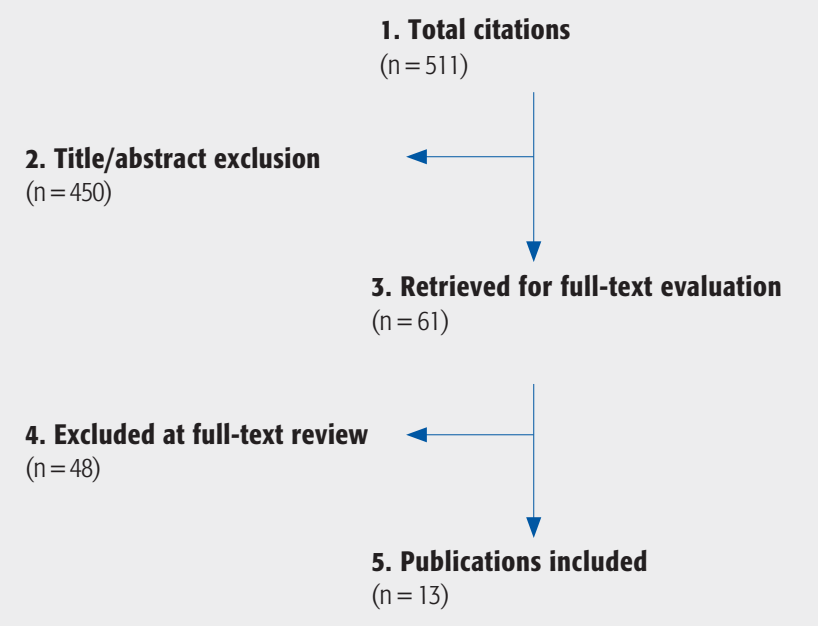

\section{MATERIALS AND METHODS}

Study design: Systematic review.

\section{Sampling:}

Search: PubMed, US Food and Drug Administration (FDA) medical device approvals and clearances. (www. fda.gov/MedicalDevices/ProductsandMedicalProcedures/DeviceApprovalsandClearances/default); bibliographies of key articles; articles citing included articles, as indexed in Google Scholar.

Dates searched: through October 2011.

\section{Inclusion criteria:}

Wear and durability assessments of artificial cervical discs retrieved from human patients and non-human animals. Biomechanical simulations of artificial cervical disc wear.

\section{Exclusion criteria:}

Finite element analyses and other mathematical modeling simulations were excluded because more direct evidence was available from retrieval and biomechanical studies, and none of the computer models had been validated physically (either with retrieved devices or in vitro biomechanical experiments).

Data reported in FDA device approvals were excluded when they appeared to be the same as those published in articles or were presented in an uninterpretable manner.

Outcomes: Wear and durability properties.

Analysis: For studies of explanted artificial cervical discs, we summarized authors' evaluations of the removed artificial discs wear, durability, and the implants' apparent effects on surrounding host tissue. We classified a wear/durability factor as present or absent based on the authors' explicit descriptions. If a factor was present, we attempted to classify the extent (density and range of dispersion) of its presence as either low or high. When authors did not indicate the extent of presence, we classified the factor as low. See the Web Appendix for details on this aspect of our analysis. For biomechanical simulation studies, we summarized results on device wear and durability as directly reported by authors.

Details on methods can be found in the electronic supplemental material at $w w w$.aospine.orglebsj 


\section{RESULTS}

- Our search yielded 511 potentially relevant citations, 61 of which we retrieved for full-text evaluation (Fig 1). Thirteen sources were ultimately included in our systematic review.

Clinical question 1: human retrieval studies

- We identified ten articles that described a total of 17 devices that had been explanted from human patients (Table 1) [1-10].

- Findings on devices' wear characteristics were not standardized across studies. Authors' reports varied in terms of the particular wear properties assessed and the methods used for such assessments. In several reports, findings were based on authors' subjective interpretations and not objective results. In some reports, the authors described wear and durability factors for some of the removed devices but not others.

- No retrieved device had been implanted more than 4 years. Time from surgery to explanation ranged from 3-39 months for the 15 cases with data on this duration.

- The Bryan cervical disc was the most commonly assessed device, with eight cases, followed by the Prestige with four cases. The KineflexlC ProDisc $C$ devices each accounted for two cases. An unnamed device was removed from another case.

- The most common indication for device removal was persistence or recurrence of preoperative symptoms (five cases). In four other cases, the devices were explanted secondary to presumed device loosening or complications of implantation. The remaining cases had unique indications for removal.

- Evidence of corrosion/oxidation was clearly present in each of the two cases (both with Prestige discs) that were evaluated on this factor. Four Bryan discs that had been implanted for periods of 4-16 months showed no evidence of oxidation or molecular weight loss in comparison with control discs from the same manufacturing lots that had not been implanted in patients [2].

- Surface abrasions were observed on the articulating surfaces in four of five cases assessed.

- Metallic debris was observed in the soft tissues around the KineflexlC (1/1 case), Prestige (3/4 cases), and Prodisc $C$ devices ( $1 / 1$ case). No metallic debris was found in the soft tissues around the Bryan device (2/2 cases) or an unnamed all-metal device with a keel.

- Polymeric debris was found in the soft tissues around each device with polymer components (2/2 Bryan and 1/l Prodisc C devices) assessed for such wear.

- Inflammatory cells were generally present to some extent in the soft tissues around the device (observed in 2/2 Bryan, 1/1 Kineflex/C, 2/3 Prestige, and 1/2 Prodisc $\mathrm{C}$ devices, and in the unnamed all-metal device with a keel). Signs of infection, however, were uncommon (found in 0/2 Bryan, 0/1 Kineflex|C, 1/3 Prestige, and $0 / 1$ Prodisc $\mathrm{C}$ devices, and not in the unnamed all-metal device with a keel).

- In one case, a ProDisc C device was explanted secondary to progressive osteolysis around the keel of the implant. In another patient, a Prestige device was loose at removal, an outcome believed to be due to excessive bone resection during the initial surgery. In another patient with a Prodisc $\mathrm{C}$ device, there was bony incorporation around the keel but not the rest of the lower end plate. In this patient, a relatively small force $(146 \mathrm{~N})$ dislodged the device from the vertebra.

- The osteolysis noted in one patient was interpreted as a reaction consistent with metal hypersensitivity. Three other patients (two with KineflexlC devices and a third with an unnamed all-metal device with a keel) had reactions that were also interpreted as a delayed hypersensitivity to metal.

Clinical question 1: non-human animal retrieval studies

- We identified three articles that described a total of 23 devices explanted from non-human animals (2 chimpanzees, 21 goats) (Table 2) [1, 5, 11].

- The time from implantation to explanation was generally shorter than in the human retrieval studies, ranging from 3-12 months.

- Bryan devices were implanted in the two chimpanzees. In these cases, there was evidence of polymeric and metallic debris and inflammation in the tissues surrounding the device, but no evidence of infection or osteolysis.

- Bryan devices were also implanted in nine goats for varying periods. As the time from implantation to explanation increased, so did the likelihood of detecting polymeric and metallic debris and signs of inflammation (rising to two of three goats with implants for 12 months).

- Porous Coated Motion devices were implanted in twelve other goats for 6 or 12 months. None of these cases showed evidence of debris, inflammation, or osteolysis.

Clinical question 2: biomechanical simulation studies

- We identified four articles that described biomechanical simulations of wear in artificial cervical discs (Table 3) $[2,8,12,13]$. The devices assessed include the Bryan, Active C, and Prestige discs.

- Simulation procedures varied, although most were conducted with devices in $37^{\circ} \mathrm{C}$ calf serum sample and all involved 10-20 million cycles at 1-4 Hz. 
Table 1 Visual, histological, and pathological findings for artificial discs retrieved from humans*

\begin{tabular}{|c|c|c|c|c|c|c|c|c|c|c|c|}
\hline ญั & : & 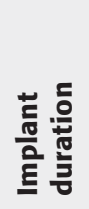 & 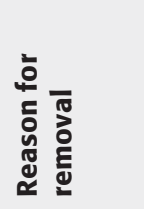 & $\frac{\text { 은 }}{\text { 은 }}$ & 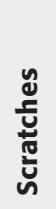 & 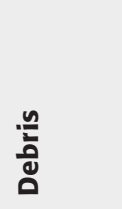 & & 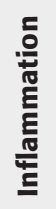 & 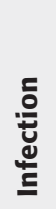 & 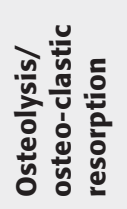 & 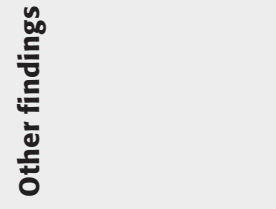 \\
\hline & & & & & & Metallic & Polymeric & & & & \\
\hline $\begin{array}{l}\text { A:Anderson et } \\
\text { al [2], }(2004)^{\dagger}\end{array}$ & Bryan & $13 \mathrm{mo}$ & $\begin{array}{l}\text { NR; device } \\
\text { did not fail }\end{array}$ & - & NR & - & + & + & - & - & \\
\hline $\begin{array}{l}\text { B: Anderson et } \\
\text { al [2] }(2004)^{\dagger}\end{array}$ & Bryan & $14 \mathrm{mo}$ & $\begin{array}{l}\text { NR; device } \\
\text { did not fail }\end{array}$ & - & NR & - & + & + & - & - & \\
\hline $\begin{array}{l}\text { C: Jensen et al } \\
\text { [5] (2005) }\end{array}$ & Bryan & $8 \mathrm{mo}$ & $\begin{array}{l}\text { Original } \\
\text { symptoms } \\
\text { ongoing }\end{array}$ & NR & NR & NR & NR & NR & NR & - & No loosening of device \\
\hline $\begin{array}{l}\text { D: Anderson et } \\
\text { al [1] (2003) }\end{array}$ & Bryan & $10 \mathrm{mo}$ & $\begin{array}{l}\text { Original } \\
\text { symptoms } \\
\text { ongoing }\end{array}$ & NR & NR & NR & NR & NR & NR & - & No loosening of device \\
\hline $\begin{array}{l}\text { E: Coric et al } \\
{[10](2011)}\end{array}$ & Kineflex'C & NR & $\begin{array}{l}\text { Persistent/ } \\
\text { recurrent } \\
\text { pain }\end{array}$ & NR & NR & NR & NA & NR & NR & NR & $\begin{array}{l}\text { Patient had delayed } \\
\text { hypersensitivity to nickel }\end{array}$ \\
\hline $\begin{array}{l}\text { F: Guyer et al } \\
\text { [4] (2011) }\end{array}$ & Kineflex'C & $14 \mathrm{mo}$ & $\begin{array}{l}\text { Presumed } \\
\text { loosening } \\
\text { of device }\end{array}$ & NR & + & ++ & NA & ++ & - & - & $\begin{array}{l}\text { Necrotic soft tissue; } \\
\text { interpreted as delayed } \\
\text { hypersensitivity to metal }\end{array}$ \\
\hline $\begin{array}{l}\text { G: Cavanaugh } \\
\text { et al [3] (2009) }\end{array}$ & $\begin{array}{l}\text { Unnamed } \\
\text { all-metal } \\
\text { device with } \\
\text { keel }\end{array}$ & $7 \mathrm{mo}$ & $\begin{array}{l}\text { Return of } \\
\text { symptoms; } \\
\text { mass near } \\
\text { spinal cord }\end{array}$ & NR & NR & - & NA & ++ & - & - & $\begin{array}{l}\text { Reaction interpreted as } \\
\text { delayed hyper-reactivity } \\
\text { to metal }\end{array}$ \\
\hline $\begin{array}{l}\text { H: Cummins et } \\
\text { al [9] (1998) }\end{array}$ & $\begin{array}{l}\text { Prestige } \\
\text { (Frenchay/ } \\
\text { Cummins) }\end{array}$ & NR & $\begin{array}{l}\text { Pain, } \\
\text { presumed } \\
\text { loosening } \\
\text { of device }\end{array}$ & NR & NR & + & NA & NR & NR & - & \\
\hline $\begin{array}{l}\text { I: Wigfield et al } \\
\text { [8] (2002) }\end{array}$ & Prestige & $12 \mathrm{mo}$ & Neck pain & NR & NR & - & NA & - & - & - & $\begin{array}{l}\text { Device was loose; authors } \\
\text { suggested too much } \\
\text { bone resected }\end{array}$ \\
\hline $\begin{array}{l}\text { J: Anderson et } \\
\text { al [2] (2004) }\end{array}$ & Prestige & $18 \mathrm{mo}$ & Infection & ++ & + & ++ & NA & ++ & ++ & - & $\begin{array}{l}\text { Much less wear than in } \\
\text { biomechanical simulation }\end{array}$ \\
\hline $\begin{array}{l}\text { K: Anderson et } \\
\text { al [2] (2004) }\end{array}$ & Prestige & $39 \mathrm{mo}$ & NR & ++ & + & ++ & NA & + & - & - & $\begin{array}{l}\text { Much less wear than in } \\
\text { biomechanical simulation }\end{array}$ \\
\hline $\begin{array}{l}\text { L: Pitzen et al } \\
\text { [6] (2007) }\end{array}$ & Prodisc C & 12 wk & Autopsy & NR & + & + & + & + & NR & - & $\begin{array}{l}\text { Bony incorporation } \\
\text { around device keel, but } \\
\text { not on device end plate; } \\
146 \mathrm{~N} \text { force required to } \\
\text { dislodge device from } \\
\text { lower end plate (much } \\
\text { less than required for } \\
\text { pulling out cervical spine } \\
\text { screws/cages) }\end{array}$ \\
\hline $\begin{array}{l}\text { M: Tumialan et } \\
\text { al [7] (2011) }\end{array}$ & Prodisc C & $15 \mathrm{mo}$ & $\begin{array}{l}\text { Progressive } \\
\text { osteoloysis }\end{array}$ & NR & - & NR & NR & - & - & + & $\begin{array}{l}\text { Osteolysis above keel, but } \\
\text { no loosening of device; } \\
\text { authors interpreted } \\
\text { reaction as consistent } \\
\text { with metal sensitivity }\end{array}$ \\
\hline
\end{tabular}

* NR indicates not reported; NA, not applicable; minus sign, not observed; plus sign, low (observed to small/modest degree; see Methods section); double plus signs, high (observed to a moderate/large degree; see Methods section).

+ Anderson et al [2] assessed four other Bryan discs for oxidation (see text), but provided no other specific information on the cases. 
Table 2 Visual, histological, and pathological findings for artificial discs retrieved from non-human animals*

\begin{tabular}{|c|c|c|c|c|c|c|c|c|c|c|c|}
\hline 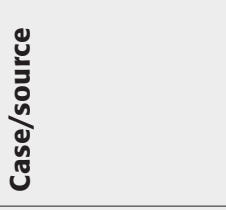 & : & 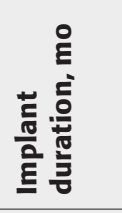 & $\begin{array}{l}\frac{y}{u} \\
\text { जे }\end{array}$ & 흔 & 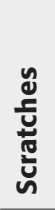 & 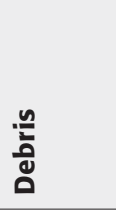 & & 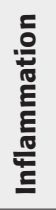 & : & 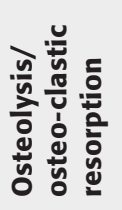 & 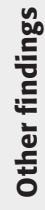 \\
\hline & & & & & & Metallic & Polymeric & & & & \\
\hline $\begin{array}{l}\text { A: Anderson et al [1] } \\
\text { (2003); Jensen et al } \\
\text { [5] (2005) }\end{array}$ & Bryan & 3 & $\begin{array}{l}\text { Chimpan- } \\
\text { zee }\end{array}$ & NR & NR & - & + & ++ & NR & - & \\
\hline $\begin{array}{l}\text { B:(Anderson et al [1] } \\
\text { (2003); Jensen et al } \\
\text { [5] (2005) }\end{array}$ & Bryan & 3 & $\begin{array}{l}\text { Chimpan- } \\
\text { zee }\end{array}$ & NR & NR & + & + & + & NR & - & \\
\hline $\begin{array}{l}\text { C: Anderson et al [1] } \\
(2003)\end{array}$ & Bryan & 3 & Goat & NR & NR & NR & - & - & NR & NR & $\dagger$ \\
\hline $\begin{array}{l}\text { D: Anderson et al [1] } \\
\text { (2003) }\end{array}$ & Bryan & 3 & Goat & NR & NR & NR & - & - & NR & NR & $\dagger$ \\
\hline $\begin{array}{l}\text { E: Anderson et al [1] } \\
(2003)\end{array}$ & Bryan & 3 & Goat & NR & NR & NR & - & - & NR & NR & $\dagger$ \\
\hline $\begin{array}{l}\text { F: Anderson et al [1] } \\
(2003)\end{array}$ & Bryan & 6 & Goat & NR & NR & NR & + & - & NR & NR & $\dagger$ \\
\hline $\begin{array}{l}\text { G: Anderson et al [1] } \\
\text { (2003) }\end{array}$ & Bryan & 6 & Goat & NR & NR & NR & - & - & NR & NR & $\dagger$ \\
\hline $\begin{array}{l}\mathrm{H}: \text { Anderson et al } 1[] \\
(2003)\end{array}$ & Bryan & 6 & Goat & NR & NR & NR & - & - & NR & NR & $\dagger$ \\
\hline $\begin{array}{l}\text { I: Anderson et al [1] } \\
(2003)\end{array}$ & Bryan & 12 & Goat & NR & NR & + & + & + & NR & NR & $\dagger$ \\
\hline $\begin{array}{l}\text { J: Anderson et al [1] } \\
(2003)\end{array}$ & Bryan & 12 & Goat & NR & NR & + & + & + & NR & NR & $t$ \\
\hline $\begin{array}{l}\text { K: Anderson et al [1] } \\
(2003)\end{array}$ & Bryan & 12 & Goat & NR & NR & - & - & - & NR & NR & $\dagger$ \\
\hline $\begin{array}{l}\text { L-W: Hu et al [11] } \\
(2006)\end{array}$ & $\begin{array}{l}\text { Porous- } \\
\text { coated } \\
\text { motion }\end{array}$ & $\begin{array}{l}6(n=6) \\
12(n=6)\end{array}$ & 12 goats & NR & NR & - & - & - & NR & - & \\
\hline
\end{tabular}

* NR indicates not reported; NA, not applicable; minus sign, not observed; plus sign, low (observed to small/modest degree; see Methods section); and double plus signs, high (observed to a moderate/large degree; see Methods section).

† No debris or abnormalities linked to the prosthesis found in lymph nodes, neural tissue, liver, or spleen. 
Table 3 Wear of artificial discs in biomechanical simulations*

\begin{tabular}{|c|c|c|c|c|c|c|c|c|}
\hline Source & Device & Simulation & $\begin{array}{l}\text { Mass } \\
\text { loss }\end{array}$ & $\begin{array}{l}\text { Height } \\
\text { loss }\end{array}$ & $\begin{array}{l}\text { Volume } \\
\text { loss }\end{array}$ & $\begin{array}{l}\text { Abra- } \\
\text { sions }\end{array}$ & $\begin{array}{l}\text { Debris } \\
\text { particles }\end{array}$ & Other findings \\
\hline $\begin{array}{l}\text { Anderson et al } \\
\text { [2] (2004) }\end{array}$ & Bryan & $\begin{array}{l}150 \mathrm{~N} \text { force flexion/extension, } \\
\text { lateral bending, and axial roation } \\
\text { in } 37^{\circ} \mathrm{C} \text { calf serum sample; } 6 \\
\text { discs: } 10 \text { million cycles bending + } \\
10 \text { million cycles rotation at } 4 \mathrm{~Hz} \text {; } \\
4 \text { discs: tested until end plate to } \\
\text { end plate contact }\end{array}$ & $\begin{array}{l}1.75 \% \text { (after } \\
20 \text { million } \\
\text { cycles in } 6 \\
\text { discs) }\end{array}$ & $\begin{array}{l}0.48 \% \text { (after } \\
20 \text { million } \\
\text { cycles in } 6 \\
\text { discs) }\end{array}$ & $\begin{array}{l}0.57 \mathrm{~mm}^{3} / \\
\text { million } \\
\text { cycles }\end{array}$ & NR & $\begin{array}{l}\text { None outside of } \\
\text { sheath; inside } \\
\text { sheath, no } \\
\text { metallic debris, } \\
\text { much polymeric } \\
\text { debris (globular } \\
\text { shape, mean } \\
\text { diameter }=4 \\
\text { microns) }\end{array}$ & $\begin{array}{l}\text { No device or } \\
\text { sheath failure } \\
\text { within } 10 \text { million } \\
\text { cycles }\end{array}$ \\
\hline $\begin{array}{l}\text { Grupp et al [13] } \\
\text { (2010) }\end{array}$ & $\begin{array}{l}\text { Active C } \\
\text { (metal on } \\
\text { polymer) }\end{array}$ & $\begin{array}{l}6 \text { discs tested with a ISO } \\
18192-1: 2008 \text { (E) } \\
\text { process: flexion, extension, } \\
\text { lateral bending, and axial roation } \\
\text { movements in sinusoidal } \\
\text { waveform with } 150 \mathrm{~N} \text { flexion } \\
\text { force and } 50 \mathrm{~N} \text { extension force, } \\
10 \text { million cycles at } 1 \mathrm{~Hz} \text { in } 37^{\circ} \mathrm{C} \\
\text { calf serum sample }\end{array}$ & $\begin{array}{l}1.0 \mathrm{mg} / \\
\text { million } \\
\text { cycles }\end{array}$ & $\begin{array}{l}0.03 \mathrm{~mm} / \\
\text { million } \\
\text { cycles for } \\
\text { polymer } \\
\text { inlay }\end{array}$ & $\begin{array}{l}1.0 \mathrm{~mm}^{3} / \\
\text { million } \\
\text { cycles }\end{array}$ & $\begin{array}{l}\text { Slight } \\
\text { scratches } \\
\text { on } \\
\text { articulating } \\
\text { surfaces }\end{array}$ & $\begin{array}{l}\text { Polymeric } \\
\text { particles were } \\
\text { numerous and } \\
\text { mostly } 0.01-1.0 \\
\text { micron in } \\
\text { diameter and } \\
\text { granular in shape } \\
\text { (mean roundness: } \\
\sim 0.5 \text { ) }\end{array}$ & $\begin{array}{l}\text { No device failure } \\
\text { reported }\end{array}$ \\
\hline FDA [12] (2009) & ProDisc C & $\begin{array}{l}10 \text { million cycles of } 150 \mathrm{~N} \text { flexion/ } \\
\text { extension, lateral bending, and } \\
\text { axial rotation at } 1 \mathrm{~Hz} \text { in } 37^{\circ} \mathrm{C} \text { calf } \\
\text { serum sample; debris sampled } \\
\text { every million cycles }\end{array}$ & $\begin{array}{l}2.59 \mathrm{mg} / \\
\text { million } \\
\text { cycles }\end{array}$ & NR & NR & NR & $\begin{array}{l}\text { Mean particle size } \\
\text { ranged from } \\
0.17-0.35 \text { microns }\end{array}$ & \\
\hline $\begin{array}{l}\text { Anderson et al } \\
\text { [2] (2004) }\end{array}$ & Prestige & $\begin{array}{l}10 \text { million cycles of } 148 \mathrm{~N} \text { flexion/ } \\
\text { extension, } 5 \text { million cycles of } 49 \\
\mathrm{~N} \text { lateral bending, and } 5 \text { million } \\
\text { cycles of } 49 \mathrm{~N} \text { axial rotation at } 2 \\
\mathrm{~Hz} \text { in } 37^{\circ} \mathrm{C} \text { calf serum sample; } \\
\text { number of discs tested not } \\
\text { reported }\end{array}$ & NR & NR & $\begin{array}{l}0.18 \mathrm{~mm}^{3} / \\
\text { million } \\
\text { cycles } \\
(0.19 \% \text { loss } \\
\text { after } 20 \\
\text { million } \\
\text { cycles })\end{array}$ & $\begin{array}{l}\text { Much more } \\
\text { wear after } \\
311,556 \\
\text { cycles than } \\
\text { in device } \\
\text { explanted } \\
\text { from a } \\
\text { patient } \\
\text { after } 39 \text { mo }\end{array}$ & NR & $\begin{array}{l}\text { No device failure } \\
\text { reported }\end{array}$ \\
\hline $\begin{array}{l}\text { Wigfield et al } \\
\text { [8] (2002) }\end{array}$ & $\begin{array}{l}\text { Prestige } \\
\text { (Frenchay) }\end{array}$ & $\begin{array}{l}10 \text { million cycles with axial loads } \\
\text { of } 150 \mathrm{~N} \text { (7 } 12 \mathrm{~mm} \text { discs) and } \\
225 \mathrm{~N} \text { (7 } 14 \mathrm{~mm} \text { disc); other tests } \\
\text { at higher forces (not reported } \\
\text { whether same or different discs) }\end{array}$ & NR & NR & NR & NR & NR & $\begin{array}{l}\text { No evidence of } \\
\text { failure at lower } \\
\text { forces; after } 5 \\
\text { million cycles at } \\
500-750 \mathrm{~N} \text {, cracks } \\
\text { near screw heads; } \\
\text { after } 120,000 \\
\text { cycles at } 1500+\mathrm{N} \text {, } \\
\text { cracks near screw } \\
\text { holes }\end{array}$ \\
\hline
\end{tabular}

*NR indicates not reported. 
- Devices were tested with forces between $49 \mathrm{~N}$ and 225 $\mathrm{N}$ depending on study and direction of loading.

- Mass and height losses were small but measures were not comparable across studies. Volume losses ranged between 0.18 and $1.0 \mathrm{~mm}^{3}$ per million cycles.

- Abrasions on the articulating surfaces of the active C and Prestige devices were slight.

- No polymeric debris escaped from the Bryan disc's sheath. The active $\mathrm{C}$ device shed many polymeric particles, most of which were 0.01-1.0 micronmeter in diameter.

- None of the devices failed in the simulations. In one study, the Prestige device sustained cracks next to screw heads at forces between 500-750 $\mathrm{N}$ and cracks in proximity to screw holes at forces greater than $1500 \mathrm{~N}$.

\section{CLINICAL GUIDELINES}

Clinical guidelines are not applicable to this topic.

\section{EVIDENCE SUMMARY}

Retrieval studies and biomechanical simulations are appropriate sources of evidence for evaluating the wear and durability of artificial cervical discs. However, conventional frameworks for classifying the strength of evidence of causal relationships between variables cannot be meaningfully applied to these types of studies in relation to the key questions our review addressed. In this context, other criteria are more useful. Notably, the included studies show generally consistent results for similar devices in both retrieval studies and biomechanical simulations. Yet the evidence is fairly limited, based on relatively few retrieved devices that had been implanted for less than 4 years. Also, evaluations of devices were often incomplete and unstandardized, and occasionally had subjective elements. Thus, it seems reasonable to classify the strength of evidence as low.

\section{DISCUSSION}

- The clinical implication of in vivo wear characteristics of C-ADR implants is not well understood and does not entirely appear to mirror the clinically significant wear seen with diarthrodial joint implants. Therefore a thorough understanding of cervical disc implant wear is critical to long-term success of the procedure.
- Polymeric debris appears to be the most common wear by-product in implants with metal on polymeric articulations (Bryan disc and ProDisc C). Each case with polymeric debris also had an inflammatory reaction in surrounding host tissue.

- Peri-implant osteolysis was not observed secondary to debris in any case. Long-term follow-up data will be needed to determine if the debris produced by the implants results in an inflammatory reaction that causes osteolysis.

- Metallic debris was noted in most devices with metal on metal articulations. Four cases of an abnormal inflammatory reaction to metal ions were reported, occurring in the Kineflex C, Prodisc C, and an unnamed all-metal device with a keel.

- The incidence of an exaggerated inflammatory response to metal ions resulting in C-ADR failure is unknown, with only a few case reports documenting its existence. However, the formation of a pseudotumor, as was the case in the Cavanaugh et al report [3], may result in compression of neural structures, making recognition of a possible metal hypersensitivity critical.

- After undergoing 10-20 million cycles of testing, no cases of outright implant failure were reported when testing was performed between 49 and $225 \mathrm{~N}$.

- The results from one study suggest that 10 million simulation cycles might approximate 50-100 years of wear [2]. Given this conclusion, the available biomechanical evidence suggests that cervical disc implants are durable under physiological loading well beyond 5 years of in vivo implantation; therefore, beyond the duration of clinical follow-up that is available.

- The following limitations should be considered in drawing conclusions from this review:

- One limitation is the small number of explanted devices available for analysis, limiting the generalizable conclusions that can be made. As more C-ADR devices are implanted and follow-up becomes longer, explanted devices for analysis will also increase.

- Many more artificial cervical discs have been explanted than have been analyzed for durability and wear. It is unknown whether devices assessed and reported in the published literature differ in some important ways from those that have not.

- No device identified was implanted more than 4 years. As a result of the short follow-up duration, long-term wear characteristics of C-ADR implants cannot be determined from this review. 


\section{CONCLUSIONS}

- Polymeric and metallic debris are produced by implant articulations in cervical discs, and are found in association with an inflammatory reaction within the host tissue. The results of this review do not suggest that particle debris causes an inflammatory reaction resulting in peri-implant osteolysis. Conclusive statements on the presence of particle-induced osteolysis will require longer follow-up data.

- Metal hypersensitivity may be a risk factor for early device failure in patients with cervical artificial disc replacement. The true prevalence and incidence of metal hypersensitivity remains unknown. Cervical artificial disc replacement should be used with caution in patients with a documented history of an abnormal inflammatory reaction to metallic implants.

- Biomechanical simulations indicate high levels of durability across tested implants with no reported device failures among the identified studies. Implants were tested from 10-20 million cycles, which has been suggested to be equivalent to 50-100 years of in vivo wear.

\section{REFERENCES}

1. Anderson PA, Rouleau JP, Bryan VE, et al (2003) Wear analysis of the Bryan Cervical Disc prosthesis. Spine (Phila Pa 1976); 28(20):S186-194.

2. Anderson PA, Rouleau JP, Toth JM, et al (2004) A comparison of simulator-tested and -retrieved cervical disc prostheses: invited submission from the Joint Section Meeting on Disorders of the Spine and Peripheral Nerves, March 2004. J Neurosurg Spine; 1(2):202-210.

3. Cavanaugh DA, Nunley PD, Kerr EJ 3rd, et al (2009) Delayed hyper-reactivity to metal ions after cervical disc arthroplasty: a case report and literature review. Spine (Phila Pa 1976); 34(7):E262-265.

4. Guyer RD, Shellock J, MacLennan B, et al (2011) Early failure of metal-on-metal artificial disc prostheses associated with lymphocytic reaction: diagnosis and treatment experience in four cases. Spine (Phila Pa 1976); 36(7):E492-497.

5. Jensen WK, Anderson PA, Nel L, et al (2005) Bone ingrowth in retrieved Bryan Cervical Disc prostheses. Spine (Phila Pa 1976); 30(22):2497-2502.

6. Pitzen T, Kettler A, Drumm J, et al (2007) Cervical spine disc prosthesis: radiographic, biomechanical and morphological post mortal findings 12 weeks after implantation: a retrieval example. Eur Spine J; 16(7):1015-1020.

7. Tumialan LM, Gluf WM (2011) Progressive vertebral body osteolysis after cervical disc arthroplasty. Spine (Phila Pa 1976); 36(14):E973-978.

8. Wigfield CC, Gill SS, Nelson RJ, et al (2002) The new Frenchay artificial cervical joint: results from a two-year pilot study. Spine (Phila Pa 1976); 27(22):2446-2452.

9. Cummins BH, Robertson JT, Gill SS (1998) Surgical experience with an implanted artificial cervical joint. J Neurosurg; 88(6):943-948.

10. Coric D, Nunley PD, Guyer RD, et al (2011) Prospective, randomized, multicenter study of cervical arthroplasty: 269 patients from the Kineflex $\mid \mathrm{C}$ artificial disc investigational device exemption study with a minimum 2-year follow-up: clinical article. J Neurosurg Spine; 15(4):348-358.

11. Hu N, Cunningham BW, McAfee PC, et al (2006) Porous coated motion cervical disc replacement: a biomechanical, histomorphometric, and biologic wear analysis in a caprine model. Spine (Phila Pa 1976); 31(15):16661673.

12. Food and Drug Administration (2009) Summary of safety and effectiveness data, ProDiscTM-C total disc replacement, P070001, December 17, 2007. Available at: www.accessdata.fda.gov/cdrh_docs/pdf7/P070001b.pdf

13. Grupp TM, Meisel HJ, Cotton JA, et al (2010) Alternative bearing materials for intervertebral disc arthroplasty. Biomaterials; 31(3):523-531. 